\title{
Machine-Executable Model-Based Systems Engineering with Graph-Based Design Languages
}

\author{
Benedikt Walter $^{1(\bowtie)}$, Dennis Kaiser ${ }^{2}$, and Stephan Rudolph ${ }^{3(\bowtie)}$ \\ ${ }^{1}$ R\&D MB Cars, Daimler AG, Sindelfingen, Germany \\ benedikt.walter@daimler.com \\ ${ }^{2}$ IILS mbH, Leinfelden, Germany \\ kaiser@iils.de \\ ${ }^{3}$ University of Stuttgart, IFB, Stuttgart, Germany \\ rudolph@ifb.uni-stuttgart.de
}

\begin{abstract}
Model-Based Systems Engineering (MBSE) structures the design process in form of a V-Model. Along the V-Model, the tasks of model creation, editing and design change propagation to maintain model consistency requires manually much time, effort and cost. While formal languages such as the Unified Modeling Language (UML) or the Systems Modeling Language (SysML) are used already to represent the design process and behavioral product aspects, the UML/SysML-models are mostly still manually assembled and interlinked. Graph-based design languages on the basis of UML combine the advantage of a digital representation of the design process with the advantage of a rule-based execution. This combination of digital representation with machineexecution boosts MBSE towards a repeatable and machine-executable V-Model. This allows for a seamless transition from manual MBSE towards an automated and machine-executable MBSE. The poster will highlight some of the advantages of this fully digital and machine-executable MBSE in an automotive dashboard application.
\end{abstract}

